\title{
Physics Must Be New Cartesian
}

\section{Dizhechko Boris Semyonowich*}

City of Sterlitamak, Bashkortostan, Russia

\section{Introduction}

The unity of physics is in the identity of space and matter. What physics avoid talking, fearing to fall into insurmountable contradictions, new Cartesian physics links simple logical connection, which is based on the assertion of Descartes that space is material and divided into infinitely small pieces. According to new Cartesian worldview of these infinitely small intervals of space are determined by the principle of certainty Heisenberg-Dizhechko and have the ability to move and rotate between them due to the virtual void. New Cartesian physics applies kinematic laws and on their basis makes conclusions that were previously impossible. In this new Cartesian Physics clearly separates geometric space from physical space. Geometric space is fixed. Physical space moves with matter that creates it [1-3].

For new Cartesian physics there is an analogue of atmospheric pressure for the entire space-matter Universe is a flow of force vector through a closed surface surrounding each interval of space. For all particles of space this thread is equal to the product of the speed of light on Planck's constant $c h$. This force acts on every particle of space and causes it to rotate and move, if there is a deviation from its stability. Due to the existence of this Law of the constancy of the flow of forces in space appear quantum properties arising from the fact that the motion of the particles begins to overcome a certain constant value of the flow of forces [2]. This Law is closely related to the determination of the mass flow through the vector of the centripetal acceleration generated by the rotation space.

New Cartesian worldview does not imply a radical revision of modern physics, as it was under theories of relativity. It only offers its reading based on the updated principle of identity of space and matter. In new Cartesian physics, there is no division of space into separate elements, as proposed by Descartes. The division of all space-matter in it is defined by a principle of irrationality. It is a principle of uncertainty of Heisenberg from quantum mechanics, transformed into the principle of definiteness of points of space Heisenberg-Dizhechko:

$$
\Delta P \geq \frac{\hbar}{\Delta x}
$$

Here, $\hbar$-Planck's constant $\mathrm{h} / 2 \pi$

In quantum mechanics the sign of the Delta is understood as a measurement error and therefore this principle it asserts the impossibility of simultaneous accurate measurements of the coordinates of the particle and its momentum, i.e. the localization of the particle at the point with coordinate ${ }_{i}$ the uncertainty in determining the magnitude of the impulse increases to infinity [4].

In new Cartesian physics, asserts the materiality of space, this inequality shows that the localization of a point in space with coordinate $x_{i}^{0}$ value increment of the pulse current in its neighborhood increases to infinity and for each increment of the impulse corresponds to a certain interval between the point with coordinate $x_{i}^{0}$ and the current point to the coordinate $x_{i}$.

$$
p_{i}-p_{i}^{0} \geq \frac{\hbar}{x_{i}-x_{i}^{0}}
$$

The larger the increment of momentum $\Delta p_{i}=p_{i}-p_{i}^{0}$ the closer the point $x_{\mathrm{i}}$ to the point $x_{i}^{0}$. In the infinitely small neighborhood of a point $x_{i}^{0}$ the increment of momentum becomes infinitely large. To have infinite momentum at the point $x_{i}^{0}$ can only be an infinitely small interval of space-matter. Because it makes no sense to talk about the angular momentum of a rational point, having neither length nor width, point $x_{i}^{0}$ irrational, meaning it is not endangered infinitely small interval, preserving at endless division and length, and breadth and thickness [5]. Therefore, in order to separate the point of space-matter from the closest points in the infinitely small interval, we need an infinite impulse, i.e. it is impossible to separate an individual object and to make the move separately from the other points. Thus, the rotation space is intervals. The angular momentum of a rotating interval of space cannot be less than Planck's constant.

The interaction of the rotating intervals of space leads to the exchange of impulses and to the continuous change of their dimensions [6-8]. This state space in contemporary physics is called the physical vacuum, which surrounds a stationary, not time-varying; threedimensional rotating its intervals, called corpuscles (particles). The corpuscles exist in the result of the fact that the pressure on them from all sides does not allow an existing in their motion dissipate in all directions. Thus, the formula of equivalence of mass and energy due to the existence pressure of the Universe and recognition of the concept of equivalence of mass and energy requires recognition of the existence of the pressure forces of the Universe:

$$
E=m c^{2} \Leftrightarrow \oint F d s=c h
$$

Right here is the formula of the law of the constancy of the flow of forces through a closed surface, which presses on the corpuscle. On the left is the formula of the law of equivalence of mass and energy particles, which counteracts the external flow forces [9]. From the fundamental law of equivalence of mass and energy, proven, should the unique versatility of the pressure forces of the Universe, which should be found in all interactions. In this case it manifests itself in the mass of the corpuscle.

$$
E=m c^{2}=\frac{c h}{2 \pi r}
$$

Where: r-the Compton wavelength.

The flow of the pressure forces of the Universe in the corpuscle is opposed to the flow of the centrifugal force:

$$
\gamma_{0} M=\oint \frac{c^{2}}{r} d s
$$

From this it follows that gravitational mass is the flow of vector of

*Corresponding author: Dizhechko Boris Semyonowich, City of Sterlitamak, Bashkortostan, Russia, Tel: 74997041341; E-mail: fizika3000@yandex.ru

Received June 02, 2017; Accepted June 30, 2017; Published July 07, 2017

Citation: Semyonowich DB (2017) Physics Must Be New Cartesian. Int J Swarm Intel Evol Comput 6: 161. doi: 10.4172/2090-4908.1000161

Copyright: @ 2017 Semyonowich DB. This is an open-access article distributed under the terms of the Creative Commons Attribution License, which permits unrestricted use, distribution, and reproduction in any medium, provided the original author and source are credited. 
the centrifugal acceleration, which is obtained if we multiply the inertial mass on the gravitational constant.

$$
\gamma_{0} M=\oint \frac{c^{2}}{r} d s
$$

Add to these formulas the factor of Lorentz, taking into account the increase of inertial mass with increasing velocity of the space, we get:

$$
\gamma_{0} M=\frac{c h}{\gamma m}
$$

A brief consideration new Cartesian physics, resulting from the clarification and development of ideas of Descartes, given the backlog in material science over the centuries since his death shows that his method makes physics not only clear, but also reveals that previously was not noticeable [7]. From the identity of space and matter it follows, that the mass is not a measure of the amount of matter and only the volume is a measure of the amount of not only space, but also substance. Mass, according to the measurement system based on two main units' length and time, is the flow of vector of centripetal acceleration during rotation of the space. To determine the mass of some region of space, we must integrate over the surface vector of the centripetal acceleration of all spins inside the body.

\section{References}

1. Bousso R (2000) Positive vacuum energy and the n-bound. J High Energy Phys 2000.

2. Bousso R, Bekenstein T (2001) Bounds in de Sitter and flat space. J High Energy Phys 2001.

3. Zlatev, Wang L, Steinhardt PJ (1998) Quintessence, cosmic coincidence and the cosmological constant. Phys Rev Lett 82: 896.

4. Hellerman S, Kaloper N, Susskind L (2001) String theory and quintessence. J High Energy Phys 2001.

5. Max B. Atomic physics. London-Glasgow.

6. http://www.wikipedia.org/wiki/Compton_wavelength

7. Zlatev, Wang L, Steinhardt PJ (1998) Quintessence, cosmic coincidence and the cosmological constant. Phys Rev Lett 82: 896.

8. Sproul RL. Modern Physics: A textbook for engineers.

9. René D (1998) The world and other writings. New York: Cambridge University Press. 